\title{
Quark Mass dependence at Two Loops for Meson Properties
}

\author{
Johan Bijnens* \\ Lund University \\ E-mail: bijnens@thep.lu.se
}

This talks contains a short introduction to Chiral Perturbation Theory and the existing calculations to two-loop order in the mesonic sector. I include a discussion on which quantities the expansion can be organized in. The present best values of the Low-Energy-Constants as determined from continuum physics are given as well as the assumptions underlying the fits to experimental data. I present plots of masses, decay constants and $f_{+}(0)$ in $K_{\ell 3}$ as a function of quark or meson masses. The talk ends with a list of things for which it would be extremely useful to have good results from lattice QCD calculations.

The XXV International Symposium on Lattice Field Theory

July 30 - August 42007

Regensburg, Germany

${ }^{*}$ Speaker. 


\section{Introduction}

An alternative title for this talk is

What is Known About Low Energy Constants and Quark Mass Dependence in Chiral Perturbation Theory from the Continuum

In order to discuss this a short introduction to Chiral perturbation Theory (ChPT) is given with an emphasis on some of the aspects that have been known to create confusion, the choice of quantities to express the expansion in. The remainder can be split in an overview of the existing calculations in two and three-flavour ChPT and quark mass dependences of several of the quantities of interest in both cases. I restrict myself here to the cases where a full two-loop calculation is available and to quantities of interest to the lattice community. I also remind you of the existing partially quenched calculations at two-loop order. In the remainder I will use order $p^{6}$ in the chiral counting, next-tonext-to-leading order (NNLO) and two-loop order as synonyms even though strictly speaking the two-loop diagrams are only part of the NNLO result.

A review of order $p^{6} \mathrm{ChPT}$ is [1] and several more references to lectures as well as files containing the long two-loop expressions can be found in the website [2]. I want to point out those by Sharpe aimed at lattice QCD practitioners [3].

\section{Chiral Perturbation Theory}

Chiral perturbation Theory in its modern form was introduced by Gasser, Leutwyler and Weinberg $[4,5,6]$ and it can be defined as

Exploring the consequences of the chiral symmetry of QCD and its spontaneous breaking using effective field theory techniques

The assumptions that are needed to allow a derivation from QCD and a full derivation can be found in the paper by Leutwyler [7]. I do not intend to give a full derivation here but only restrict myself to a few comments.

Powercounting: In effective field theory, one assumes that there is a gap in the spectrum which allows to include only the degrees of freedom that are relevant below the gap and treat the effects of the degrees of freedom at higher scales perturbatively. Thus a clear separation of scales is the first requirement. One then constructs the most general local Lagrangian with the lower degrees of freedom in agreement with the symmetries and their realization. Unfortunately, this leads to an infinite number of parameters and hence there is no predictivity left. If there exists a way to organize the series in terms of order of importance, then we can work order by order and have predictivity at any fixed order in the importance. This is typically achieved by introducing powercounting.

Unitarity: In Ref. [7] one uses strongly the fact that the only singularities at low energies in Green functions come from poles and cuts of the light degrees of freedom and that all the remaining vertices can be expanded. This is where unitarity plays a role in the derivation of ChPT from QCD.

ChPT: ChPT is the effective field theory built with the Goldstone Bosons resulting from the spontaneous breakdown of chiral symmetry in QCD as primary degrees of freedom. The powercounting used ${ }^{1}$ is basically dimensional counting in momenta and (meson) masses. The expected

\footnotetext{
${ }^{1}$ There are different countings possible, the one here is the standard one. References can be found in [1] and the
} 
breakdown scale is the scale of resonances, so for energies around $m_{\rho}$ standard mesonic ChPT definitely does not work. The breakdown scale depnds a bit on the channel.

Chiral Symmetry: In QCD with three light quarks of equal mass, they are fully interchangeable and we have a $S U(3)_{V}$ symmetry. But looking at the QCD Lagrangian

$$
\mathscr{L}_{Q C D}=\sum_{q=u, d, s}\left[i \bar{q}_{L} \not \supset q_{L}+i \bar{q}_{R} \not D q_{R}-m_{q}\left(\bar{q}_{R} q_{L}+\bar{q}_{L} q_{R}\right)\right]
$$

we see that for $m_{q}=0$ we have a separate interchange for the left and right-handed quarks: $G_{\chi}=$ $S U(3)_{L} \times S U(3)_{R}$.

Chiral Symmetry Breaking: The fact that the vacuum expectation value $\langle\bar{q} q\rangle=\left\langle\bar{q}_{L} q_{R}+\bar{q}_{R} q_{L}\right\rangle \neq$ 0 leads to the spontaneous breaking of $S U(3)_{L} \times S U(3)_{R}$ to $S U(3)_{V}$. The eight broken generators lead to eight massless degrees of freedom and make their interactions vanish at zero momentum. The latter fact allows to produce a consistent powercounting in ChPT. This is illustrated in Fig. 1.
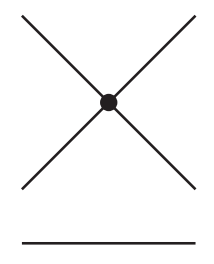

$\int d^{4} p$

$$
p^{2}
$$$$
1 / p^{2}
$$

$p^{4}$

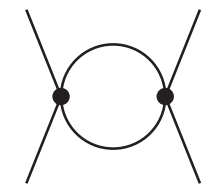

$$
\left(p^{2}\right)^{2}\left(1 / p^{2}\right)^{2} p^{4}=p^{4}
$$

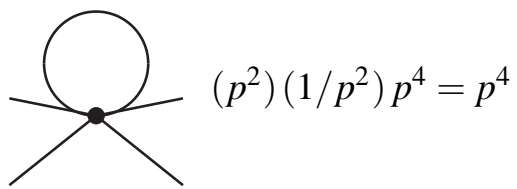

Figure 1: An illustration of the power-counting in ChPT. On the left we see the lowest order vertex with two powers of momenta or masses, the meson propagator with two inverse powers and the loop integration leading to four powers. On the right-hand side we see two one-loop contributions and how the counting on the left leads to the same power $p^{4}$ for both diagrams. This counting was generalized to all orders in [4].

Chiral Perturbation Theories: ChPT is a very large subject, more than 3500 papers cite at least one of the three basic papers. The name ChPT also is given to a variety of different theories exploiting the chiral symmetry of QCD. These include application to baryons, mesons and baryons containing heavy quarks, vector mesons and other resonances and of course the light pseudoscalar mesons. Within the latter we can distinguish between theories with two, three or more flavours, also in the partially quenched varieties, as well as including electromagnetism and the weak interactions nonleptonically and the possibility of treating the kaon as a heavy particle. This talk restricts itself to the standard two, three or more flavour sector with strong interactions and couplings to external currents. This is the part that has been most fully pushed to order $p^{6}$.

Lowest order Lagrangian: The Goldstone Bosons live on the manifold $G / H$ with $G=S U\left(n_{F}\right)_{L} \times$ $S U\left(n_{F}\right)_{R}$ and $H=S U\left(n_{F}\right)_{V}$ for $n_{F}$ flavours of quarks. $G / H$ has again the structure of $S U\left(n_{F}\right)$ and can be parametrized by a matrix

$$
U(\phi)=\exp \left(i \sqrt{2} \Phi / F_{0}\right), \quad \text { with } \quad \Phi(x)=\left(\begin{array}{ccc}
\frac{\pi^{0}}{\sqrt{2}}+\frac{\eta_{8}}{\sqrt{6}} & \pi^{+} & K^{+} \\
\pi^{-} & -\frac{\pi^{0}}{\sqrt{2}}+\frac{\eta_{8}}{\sqrt{6}} & K^{0} \\
K^{-} & \bar{K}^{0} & -\frac{2 \eta_{8}}{\sqrt{6}}
\end{array}\right) .
$$

talk by S. Descotes-Genon[8]. 


\begin{tabular}{ccccccc} 
& \multicolumn{2}{c}{2 flavour } & \multicolumn{2}{c}{3 flavour } & \multicolumn{2}{c}{$3+3$ PQChPT } \\
\hline$p^{2}$ & $F, B$ & 2 & $F_{0}, B_{0}$ & 2 & $F_{0}, B_{0}$ & 2 \\
$p^{4}$ & $l_{i}^{r}, h_{i}^{r}$ & $7+3$ & $L_{i}^{r}, H_{i}^{r}$ & $10+2$ & $\hat{L}_{i}^{r}, \hat{H}_{i}^{r}$ & $11+2$ \\
$p^{6}$ & $c_{i}^{r}$ & $52+4$ & $C_{i}^{r}$ & $90+4$ & $K_{i}^{r}$ & $112+3$ \\
\hline
\end{tabular}

Table 1: The number of parameters+contact terms for the various types of ChPT.

Here I have indicated the traceless matrix $\Phi$ in terms of the known pseudoscalars for the case of $n_{F}=3$. The lowest order or order $p^{2}$ Lagrangian contains two terms

$$
\mathscr{L}_{2}=\left(F_{0}^{2} / 4\right)\left\{\left\langle D_{\mu} U^{\dagger} D^{\mu} U\right\rangle+\left\langle\chi^{\dagger} U+\chi U^{\dagger}\right\rangle\right\},
$$

with a covariant derivative $D_{\mu} U=\partial_{\mu} U-i r_{\mu} U+i U l_{\mu}$, which includes the left and right external currents: $r(l)_{\mu}=v_{\mu}+(-) a_{\mu}$. The scalar and pseudoscalar external densities are included via $\chi=2 B_{0}(s+i p)$ with the quark masses included in the scalar density: $s=\mathscr{M}+\cdots$. The notation $\langle A\rangle$ is the trace over flavours $\operatorname{Tr}_{F}(A)$.

NLO Lagrangian: The Lagrangian at order $p^{4}$ was classified in $[5,6]$ and is for $n_{F}=3$

$$
\begin{aligned}
\mathscr{L}_{4}= & L_{1}\left\langle D_{\mu} U^{\dagger} D^{\mu} U\right\rangle^{2}+L_{2}\left\langle D_{\mu} U^{\dagger} D_{v} U\right\rangle\left\langle D^{\mu} U^{\dagger} D^{v} U\right\rangle+L_{3}\left\langle D^{\mu} U^{\dagger} D_{\mu} U D^{v} U^{\dagger} D_{v} U\right\rangle \\
& +L_{4}\left\langle D^{\mu} U^{\dagger} D_{\mu} U\right\rangle\left\langle\chi^{\dagger} U+\chi U^{\dagger}\right\rangle+L_{5}\left\langle D^{\mu} U^{\dagger} D_{\mu} U\left(\chi^{\dagger} U+U^{\dagger} \chi\right)\right\rangle+L_{6}\left\langle\chi^{\dagger} U+\chi U^{\dagger}\right\rangle^{2} \\
& +L_{7}\left\langle\chi^{\dagger} U-\chi U^{\dagger}\right\rangle^{2}+L_{8}\left\langle\chi^{\dagger} U \chi^{\dagger} U+\chi U^{\dagger} \chi U^{\dagger}\right\rangle-i L_{9}\left\langle F_{\mu \nu}^{R} D^{\mu} U D^{v} U^{\dagger}+F_{\mu v}^{L} D^{\mu} U^{\dagger} D^{v} U\right\rangle \\
& +L_{10}\left\langle U^{\dagger} F_{\mu v}^{R} U F^{L \mu v}\right\rangle+H_{1}\left\langle F_{\mu v}^{R} F^{R \mu v}+F_{\mu v}^{L} F^{L \mu v}\right\rangle+H_{2}\left\langle\chi^{\dagger} \chi\right\rangle .
\end{aligned}
$$

The constants $L_{i}$ in this Lagrangian are generally known as Low-energy constants (LECs). The constants $H_{i}$ have values dependent on the definition of currents/densities and the terms are called contact terms. The LECs absorb the divergences of loop diagrams order by order in the powercounting. The finite part is denoted by $L_{i}^{r}$ and depends on the subtraction scale $\mu$ and the renormalization prescription.

Number of parameters: The number of parameters at the various orders is shown in Tab. 1. The order $p^{2}$ is from [9], order $p^{4}$ from [5, 6], order $p^{6}$ from [10] after an earlier attempt [11]. The partially quenched results are derived from the $n_{F}$ flavour case $[12,13]$. The difficulty in obtaining a minimal set can be seen from the recent discovery of a new relation in the $n_{F}=2$ case [14]. Since the normal case is a continuous limit of the partially quenched case, the resulting LECs are just linear combinations of partially quenched LECs using the Cayley-Hamilton relations given in [10]. The general divergence structure at this order is also known [15]. The parameters $B \neq B_{0}$ and $F \neq F_{0}$ are the two versus three-flavour lowest order constants.

Chiral Logarithms: The main predictions of ChPT are twofold. 1) It relates processes with different numbers of pseudoscalars. 2) It predicts nonanalytic dependences at higher orders, often referred to generically as Chiral $\log ($ arithm $) s$. As an example, the pion mass for $n_{F}=2$ is given at NLO by [5]

$$
m_{\pi}^{2}=2 B \hat{m}+\left(\frac{2 B \hat{m}}{F}\right)^{2}\left[\frac{1}{32 \pi^{2}} \log \frac{(2 B \hat{m})}{\mu^{2}}+2 l_{3}^{r}(\mu)\right]+\cdots
$$

The notation $M^{2}=2 B \hat{m} \equiv B\left(m_{u}+m_{d}\right)$ is used a lot in the remainder. The $\mu$ dependence cancels between the explicit dependence in the logarithm and the implicit dependence in $l_{3}^{r}$. 
LECs and choice of $\mu$ : The LECs, like $l_{3}^{r}$ in (2.5), have to be determined experimentally or from lattice calculations. They can be quoted in several ways. For $n_{F}=2$ Ref. [5] introduced

$$
\bar{l}_{i}=\left(32 \pi^{2} / \gamma_{i}\right) l_{i}^{r}(\mu)-\log \left(m_{\pi}^{2} / \mu^{2}\right)
$$

which are $\mu$ independent and are proportional to the LECs $l_{i}^{r}\left(\mu=m_{\pi}\right)$. For $n_{F}=3$ some of the corresponding $\gamma_{i}$ are zero and no good equivalent definition of $\bar{L}_{i}$ exists. Here we always quote the $L_{i}^{r}(\mu)$. The scale $\mu$ is in principle arbitrary but becomes relevant when using estimates for higher order constants. Choosing $\mu=m_{\pi}, m_{K}$ or $m_{\eta}$ puts some of the chiral logs to zero and thus obscures one of the main predictions of ChPT. At a scale $\mu \approx 1 \mathrm{GeV}$ experimentally $L_{5}^{r} \approx 0$ and this would clash with large $N_{c}$ type estimates of the LECs. For these reasons, many ChPT practitioners use $\mu=m_{\rho}=0.77 \mathrm{GeV}$.

What quantities to expand in: The ChPT expansion is in momenta and masses. However, one first has to decide whether to expand in lowest order quantities, like $F, 2 B \hat{m}$, or physical masses and decay constants, like $m_{\pi}, m_{K}, m_{\eta}, F_{\pi}, F_{K}$. The latter is also not unique since relations like the Gell-Mann-Okubo relation and kinematical relations like $s+t+u=2 m_{\pi}^{2}+2 m_{K}^{2}$ for $\pi K$-scattering can be (and are heavily) used to rewrite expressions. This sounds trivial but can change much how a series convergence looks as shown below for a simple example. Similar questions are also discussed by [8]. I personally prefer to use physical masses and decay constants rather than the lowest order quantities. The physical quantities are typically better known and the chiral logs are created by particles propagating with their physical momentum. Also, thresholds appear in the right places at each order in perturbation theory.

A very simple example: Take the relations $m_{\pi}=m_{0} /\left(1+a m_{0} / f_{0}\right), \quad f_{\pi}=f_{0} /\left(1+b m_{0} / f_{0}\right)$, as exact. We can expand to NNLO in several ways

$$
\begin{array}{cc}
m_{\pi}=m_{0}-a \frac{m_{0}^{2}}{f_{0}}+a^{2} \frac{m_{0}^{3}}{f_{0}^{2}}+\cdots & f_{\pi}=f_{0}\left(1-b \frac{m_{0}}{f_{0}}+b^{2} \frac{m_{0}^{2}}{f_{0}^{2}}+\cdots\right) \\
m_{\pi}=m_{0}-a \frac{m_{\pi}^{2}}{f_{\pi}}+a(b-a) \frac{m_{\pi}^{3}}{f_{\pi}^{2}}+\cdots & f_{\pi}=f_{0}\left(1-b \frac{m_{\pi}}{f_{\pi}}+b(2 b-a) \frac{m_{\pi}^{2}}{f_{\pi}^{2}}+\cdots\right) \\
m_{\pi}=m_{0}\left(1-a \frac{m_{\pi}}{f_{\pi}}+a b \frac{m_{\pi}^{2}}{f_{\pi}^{2}}+\cdots\right) &
\end{array}
$$

The coefficients in the expansion and the actual numerical values clearly depend on the way we write the results. The plots in Fig. 2 show the convergence for $a=1, b=0.5$ and $f_{0}=1$. Only knowing the first three terms one would draw very different conclusions on the quality of the convergence from Fig. 2 for the different ways of writing the expansion.

\section{Two-flavour ChPT at NNLO}

References to order $p^{2}$ and $p^{4}$ work can be found in [1]. The first work at NNLO used dispersive methods to obtain the nonanalytic dependence on kinematical quantities, $q^{2}, s, t, u$ at NNLO. This was done for the vector (electromagnetic) and scalar formfactor of the pion in [16] (numerically) and [17] (analytically) and for $\pi \pi$-scattering analytically in [18]. The work of [18] allowed to put many of the full NNLO ChPT calculations in two-flavour ChPT in a simple analytical form. 

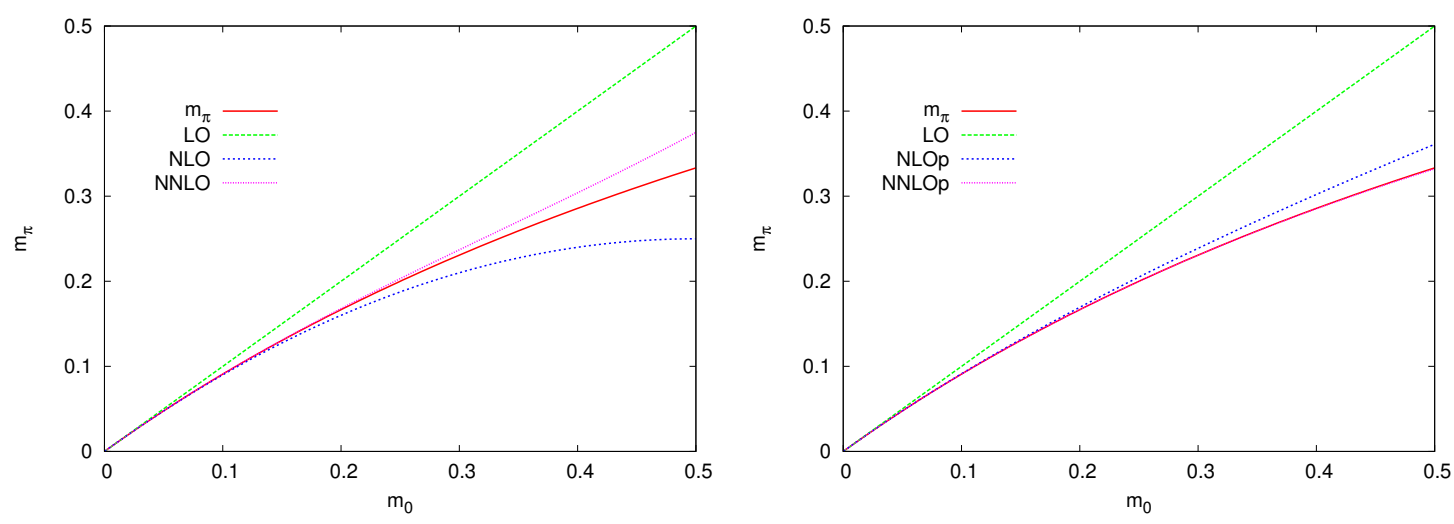

Figure 2: On the left $m_{\pi}$ as a function of $m_{0}$ for the expansion in terms of $m_{o} / f_{0}(2.7)$ and on the right for the expansion in terms of $m_{\pi} / f_{\pi}(2.8)$. Shown are the full results $\left(m_{\pi}\right)$ and the first three approximations.

Essentially all processes of interest are calculated to NNLO fully in ChPT starting with $\gamma \gamma \rightarrow$ $\pi^{0} \pi^{0}[19,20], \gamma \gamma \rightarrow \pi^{+} \pi^{-}$[21, 22, 23], $F_{\pi}$ and $m_{\pi}$ [22, 24, 25, 26], $\pi \pi$-scattering [24, 25], the pion scalar and vector formfactors [26] and pion radiative decay $\pi \rightarrow \ell v \gamma$ [27]. The pion mass is also known at order $p^{6}$ in finite volume [28].

The LECs have been fitted in several processes. $\bar{l}_{4}$ from fitting to the pion scalar radius [27, 29], $\bar{l}_{3}$ from an estimate of the pion mass dependence on the quark masses [5, 29] and $\bar{l}_{1}, \bar{l}_{2}$ from the agreement with $\pi \pi$-scattering [29], $\bar{l}_{6}$ from the pion charge radius [26] and $\bar{l}_{6}-\bar{l}_{5}$ from the axial formfactor in $\pi \rightarrow \ell v \gamma$. The final best values are [26, 27, 29]

$$
\begin{array}{lll}
\bar{l}_{1}=-0.4 \pm 0.6, & \bar{l}_{2}=4.3 \pm 0.1, & \bar{l}_{3}=2.9 \pm 2.4 \\
\bar{l}_{4}=4.4 \pm 0.2, & \bar{l}_{6}-\bar{l}_{5}=3.0 \pm 0.3, & \bar{l}_{6}=16.0 \pm 0.5 \pm 0.7 .
\end{array}
$$

It should be noticed that we do not have a good determination of $\bar{l}_{3}$ from the continuum.

There is also information on some combinations of $p^{6}$ LECs. These are basically via the curvature in the vector and scalar formfactor of the pion [26] and two combinations from $\pi \pi$ scattering [29] from the knowledge of $b_{5}$ and $b_{6}$ in that reference. The order $p^{6}$ LECs $c_{i}^{r}$ are estimated to have a small effect for $m_{\pi}, f_{\pi}$ and $\pi \pi$-scattering.

Let me now show a few dependences on the quark mass via $M^{2}=2 B \hat{m}$. First for $m_{\pi}^{2}$ expanded in analogy with (2.9). A surprise is how small the NLO and NNLO corrections are for the values of the input parameters given in (3.1) and $c_{i}^{r}(\mu=0.77 \mathrm{GeV})=0$. The full result is extremely linear as can be seen in the left plot in Fig. 3 The linearity is a consequence of the fitting parameters as can be seen in the right figure in Fig. 3. Similarly, $F_{\pi}$ as a function of $M^{2}$ expanded as in (2.8) is shown in Fig. 4. The values of $m_{\pi}^{2}, F_{\pi}$ and $M^{2}$ are determined selfconsistently from the ChPT formulas quoted in [26] via an iterative method.

\section{Three-flavour ChPT at NNLO}

\subsection{Calculations}

In this section I will discuss several results at NNLO in mesonic three-flavour ChPT. In general the formulas here are much more involved than in two-flavour ChPT and while the expressions have 

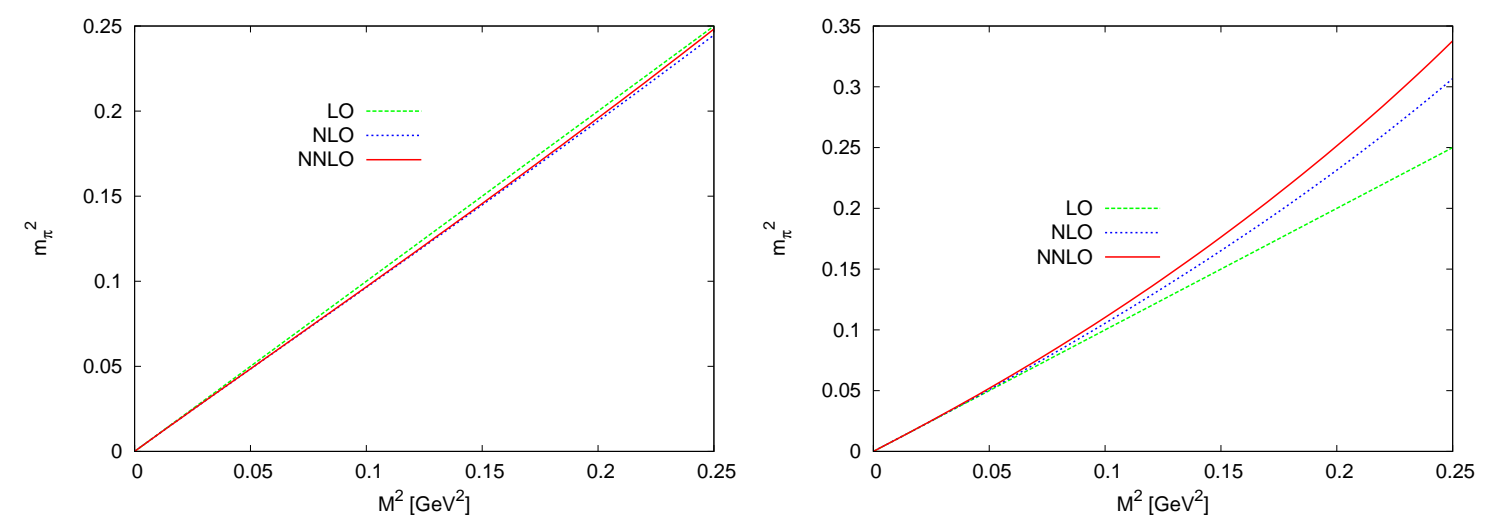

Figure 3: The pion mass squared as a function of the quark mass via $M^{2}=2 B \hat{m}$, left with inputs as in (3.1) and right with $\bar{l}_{3}=0$, both are for $n_{F}=2 \mathrm{ChPT}$.

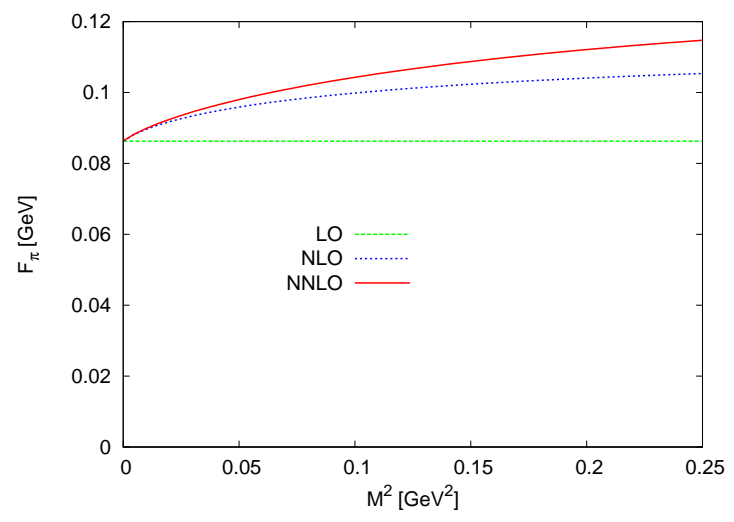

Figure 4: The pion decay constant as a function of the quark mass via $M^{2}=2 B \hat{m}$, for $n_{F}=2$ ChPT.

been reduced to a series of well-defined two-loop integrals, the latter are evaluated numerically. Most recent calculations use the subtraction scheme specified in $[15,25]$ but many do not, also the reduction to numerical integrals is done differently by different groups which makes comparisons very difficult in the case of disagreement, see e.g. the discussion in [30] about the numerical discrepancy with [31].

The vector two-point functions were among the first calculated in the $\pi$ and $\eta$ flavour quantum number channel [32,33] and in the $K$ flavour quantum number channel $[32,34]$. The isospin breaking for the $\rho \omega$ channel was done in [35]. The disconnected scalar two-point function relevant for bounds on $L_{4}^{r}$ and $L_{6}^{r}$ was worked out in [36]. The remaining scalar two-point functions are known but unpublished [37]. Masses and decay constants as well as axial-vector two-point functions were the first calculations which required full two-loop integrals, done in the $\pi$ and $\eta[32,38]$ and the $K$ channel [32]. The full isospin breaking contributions to masses and decay constants are in [39].

At this level many expressions were known but a full fit of LECs to experimental data could be carried out only after $K_{\ell 4}$ had also been evaluated to NNLO [40, 41]. The vacuum expectation values in the isospin limit were done in [41], with isospin breaking in [39] and at finite volume in [42]. 
The vector (electromagnetic) formfactors for pions and kaons were calculated in [43, 44, 45] and in [45] a NNLO fit for $L_{9}^{r}$ was performed. $L_{10}^{r}$ can be determined from the axial formfactor in $\pi, K \rightarrow \ell v \gamma$. The NNLO calculation is done, but no data fitting was performed[46]. A rather important calculation is the $K_{\ell 3}$ formfactor. This calculation was done by [30,31] and a rather interesting relation between the value at zero, the slope and the curvature for the scalar formfactor obtained [30]. I will present some results for $K_{\ell 3}$ below.

Calculations for scalar formfactors including sigma terms and scalar radii [47] and $\pi \pi$ [48] and $\pi K$-scattering [49] have been performed as well and used to place limits on $L_{4}^{r}$ and $L_{6}^{r}$. Finally, the relations between the $l_{i}^{r}$ and $L_{i}^{r}$ have been extended to the accuracy needed to compare order $p^{6}$ results in two and three-flavour calculations [50] and there has been some progress towards fully analytical results for $m_{\pi}^{2}$ [51] and $\pi K$-scattering lengths [52].

I am aware of a number of calculations in progress, including $\eta \rightarrow 3 \pi$ and isospin breaking in $K_{\ell 3}$ [53], preliminary results were reported in [54], the sunsetintegrals needed for the masses at finite volume [55] and the relations between the two and three flavour order $p^{6}$ constants [56].

\section{2 $C_{i}^{r}$ : estimates of order $p^{6}$ LECs}

Most numerical analyses at order $p^{6}$ use a (single) resonance approximation to the order $p^{6}$ LECs. This is schematically shown in Fig. 5. The main underlying motivation is the large $N_{c}$ limit
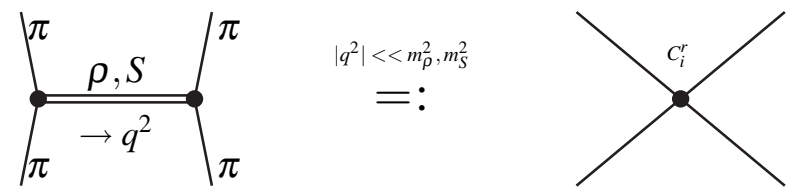

Figure 5: A schematic indication of the estimate of the order $p^{6}$ LECs by resonance exchange.

and phenomenological success at order $p^{4}$ [57]. There is a large volume of work on this, some references are $[58,59,60,61]$. The numerical work I will report has used the simple resonance Lagrangian [25, 39, 41, 57]

$$
\begin{aligned}
\mathscr{L}_{V} & =-\frac{1}{4}\left\langle V_{\mu \nu} V^{\mu v}\right\rangle+\frac{1}{2} m_{V}^{2}\left\langle V_{\mu} V^{\mu}\right\rangle-\frac{f_{V}}{2 \sqrt{2}}\left\langle V_{\mu v} f_{+}^{\mu v}\right\rangle-\frac{i g_{V}}{2 \sqrt{2}}\left\langle V_{\mu v}\left[u^{\mu}, u^{v}\right]\right\rangle+f_{\chi}\left\langle V_{\mu}\left[u^{\mu}, \chi_{-}\right]\right\rangle, \\
\mathscr{L}_{A} & =-\frac{1}{4}\left\langle A_{\mu v} A^{\mu v}\right\rangle+\frac{1}{2} m_{A}^{2}\left\langle A_{\mu} A^{\mu}\right\rangle-\frac{f_{A}}{2 \sqrt{2}}\left\langle A_{\mu v} f_{-}^{\mu v}\right\rangle, \\
\mathscr{L}_{S} & =\frac{1}{2}\left\langle\nabla^{\mu} S \nabla_{\mu} S-m_{S}^{2} S^{2}\right\rangle+c_{d}\left\langle S u^{\mu} u_{\mu}\right\rangle+c_{m}\left\langle S \chi_{+}\right\rangle, \mathscr{L}_{\eta^{\prime}}=\frac{1}{2} \partial_{\mu} P_{1} \partial^{\mu} P_{1}-\frac{1}{2} m_{\eta^{\prime}}^{2} P_{1}^{2}+i \tilde{d}_{m} P_{1}\left\langle\chi_{-}\right\rangle . \\
f_{V} & =0.20, \quad f_{\chi}=-0.025, \quad g_{V}=0.09, \quad c_{m}=42 \mathrm{MeV}, \quad c_{d}=32 \mathrm{MeV}, \quad \tilde{d}_{m}=20 \mathrm{MeV}, \\
m_{V} & =m_{\rho}=0.77 \mathrm{GeV}, m_{A}=m_{a_{1}}=1.23 \mathrm{GeV}, m_{S}=0.98 \mathrm{GeV}, \quad m_{P_{1}}=0.958 \mathrm{GeV} .
\end{aligned}
$$

The values of $f_{V}, g_{V}, f_{\chi}$ and $f_{A}$ come from experiment [25, 57] and $c_{m}$ and $c_{d}$ from resonance saturation at order $p^{4}$ [57].

The estimate of the $C_{i}^{r}$ is the weakest point in the numerical fitting at present, however, many results are not very sensitive to this. The main problem is how the $C_{i}^{r}$ which contribute to the masses, estimated to be zero except for $\eta^{\prime}$ effects by (4.1), affect the determination of the others. The estimate is also $\mu$-independent while the $C_{i}^{r}$ depend on $\mu$. 
The fits done here in $[39,40,41,47]$ try to check this by varying the total resonance contribution by a factor of two, varying the scale $\mu$ from 550 to $1000 \mathrm{MeV}$ and compare estimated $C_{i}^{r}$ to experimentally determined ones. The latter works well, but again the experimentally well determined ones are those with dependence on kinematic variables only, not ones relevant for quark-mass dependence.

\subsection{The fitting and results}

The inputs used for the fitting, see the more extensive discussion in [39, 41], are

- $K_{\ell 4}: F(0), G(0), \lambda$ from E865 at BNL[62, 63].

- $m_{\pi^{0}}^{2}, m_{\eta}^{2}, m_{K^{+}}^{2}, m_{K^{0}}^{2}$, electromagnetic corrections include the estimated violation of Dashen's theorem ([64] and references therein).

- $F_{\pi^{+}}$.

- $F_{K^{+}} / F_{\pi^{+}}$.

- $m_{s} / \hat{m}=24$. Variations with $m_{s} / \hat{m}$ were studied in $[39,41]$.

- $L_{4}^{r}, L_{6}^{r}$ the main fit, 10 , has them equal to zero, but see below and the arguments in [36].

The results of this fit are summarized in Tab. 2. The errors are very correlated, this is shown in Fig. 6 in [41] for the fit to then available $K_{\ell 4}$ data. As said before varying the estimates of the $C_{i}^{r}$ by a factor of two, varying the $\mu$ where the estimate of the $C_{i}^{r}$ is done from 550 to $1000 \mathrm{MeV}$ all stay within the given errors. Varying the values of $L_{4}^{r}, L_{6}^{r}$ as input can be done with a reasonable fitting chi-squared when varying $10^{3} L_{4}^{r}$ from -0.4 to 0.6 and $L_{6}^{r}$ from -0.3 to 0.6 . These alternative fits were performed in [47] and variation of many quantities with $L_{4}^{r}, L_{6}^{r}$ (including the changes via the changed values of the other $L_{i}^{r}$ ) are shown in [47, 48, 49]. Fit B was one of the fits that gave a good fit to the pion scalar radius and fairly small corrections to the sigma terms [47] while fit D [65] is the one that gave agreement with $\pi \pi$ and $\pi K$-scattering threshold quantities.

One point should be observed here, if one fits lattice data with NLO formulas to obtain the $L_{i}^{r}$, one should also use the NLO or order $p^{4}$ fit values from Tab. 2 to compare with.

Note the $m_{u} / m_{d}=0$ is never even close to the best fit and this remains true for the entire variation with $L_{4}^{r}, L_{6}^{r}$. The value of $F_{0}$, the pion decay constant in the three-flavour chiral limit, can vary significantly, even though I believe that fit $\mathrm{B}$ is an extreme case.

In Fig. 6 we show how the threshold parameters $a_{0}^{0}$ and $a_{0}^{2}$ depend on the variation with $L_{4}^{r}, L_{6}^{r}$. $a_{0}^{0}$ always agrees well with the result of [29] while $a_{0}^{2}$ only agrees well within a limited region [48]. For comparison, the order $p^{2}$ values are $a_{0}^{0}=0.159$ and $a_{0}^{2}=-0.0454$. The planes in Fig. 6 indicate the results $a_{0}^{0}=0.220 \pm 0.005, a_{0}^{2}=-0.0444 \pm 0.0010$ [29]. The same study was performed for $\pi K$ scattering lengths in [49] with the results of the Roy-Steiner analysis [66]. The resulting limits on the input values of $L_{4}^{r}, L_{6}^{r}$ are shown in Fig. 7. The resulting region called fit D in Tab. 2 is $10^{3} L_{4}^{r} \approx 0.2,10^{3} L_{6}^{r} \approx 0.0$. This general fitting obviously needs more work and systematic studies and constraints from lattice QCD on $L_{4}^{r}, L_{6}^{r}$ will be very useful.

\subsection{Mass dependence and other results for selected quantities}

I now show the dependence of a few quantities on the input masses. These are updates of the plots shown in [41] and also some new ones for $f_{+}(0)$ in $K_{\ell 3}$. The masses squared and decay 


\begin{tabular}{ccccc} 
& fit 10 & same $p^{4}$ & fit B & fit D \\
\hline $10^{3} L_{1}^{r}$ & $0.43 \pm 0.12$ & 0.38 & 0.44 & 0.44 \\
$10^{3} L_{2}^{r}$ & $0.73 \pm 0.12$ & 1.59 & 0.60 & 0.69 \\
$10^{3} L_{3}^{r}$ & $-2.35 \pm 0.37$ & -2.91 & -2.31 & -2.33 \\
$10^{3} L_{4}^{r}$ & $\equiv 0$ & $\equiv 0$ & $\equiv 0.5$ & $\equiv 0.2$ \\
$10^{3} L_{5}^{r}$ & $0.97 \pm 0.11$ & 1.46 & 0.82 & 0.88 \\
$10^{3} L_{6}^{r}$ & $\equiv 0$ & $\equiv 0$ & $\equiv 0.1$ & $\equiv 0$ \\
$10^{3} L_{7}^{r}$ & $-0.31 \pm 0.14$ & -0.49 & -0.26 & -0.28 \\
$10^{3} L_{8}^{r}$ & $0.60 \pm 0.18$ & 1.00 & 0.50 & 0.54 \\
$10^{3} L_{9}^{r}$ & $5.93 \pm 0.43$ & 7.0 & - & - \\
\hline $2 B_{0} \hat{m} / m_{\pi}^{2}$ & 0.736 & 0.991 & 1.129 & 0.958 \\
$m_{\pi}^{2}: p^{4}, p^{6}$ & $0.006,0.258$ & $0.009, \equiv 0$ & $-0.138,0.009$ & $-0.091,0.133$ \\
$m_{K}^{2}: p^{4}, p^{6}$ & $0.007,0.306$ & $0.075, \equiv 0$ & $-0.149,0.094$ & $-0.096,0.201$ \\
$m_{\eta}^{2}: p^{4}, p^{6}$ & $-0.052,0.318$ & $0.013, \equiv 0$ & $-0.197,0.073$ & $-0.151,0.197$ \\
$m_{u} / m_{d}$ & $0.45 \pm 0.05$ & 0.52 & 0.52 & 0.50 \\
\hline$F_{0}[\mathrm{MeV}]$ & 87.7 & 81.1 & 70.4 & 80.4 \\
$\frac{F_{K}}{F_{\pi}}: p^{4}, p^{6}$ & $0.169,0.051$ & $0.22, \equiv 0$ & $0.153,0.067$ & $0.159,0.061$
\end{tabular}

Table 2: The fits of the $L_{i}^{r}$ and some results, see text for a detailed description. They are all quoted at $\mu=0.77 \mathrm{GeV}$. Table with values from [39, 45, 47, 49, 65]. At present the best fits to use for comparison with the lattice are fit 10 at NNLO or order $p^{4}$ depending on the order of the lattice fit.

constants are written in the form analogous to (2.8) as published in [32] (note the erratum of [39] and the formulas given in [2]). A selfconsistent set of $m_{\pi}^{2}, m_{K}^{2}, m_{\eta}^{2}, F_{\pi}, B_{0} m_{s}$ and $B_{0} \hat{m}$ with the fitted values of $L_{i}^{r}$ and $F_{0}$ is determined for each input value of two masses. This is done by iterating the formulas till convergence is reached. NNLO reproduces the physical values at the physical point.

We show $m_{\pi}^{2}$ for fit 10 and fit $\mathrm{D}$ keeping $m_{s} / \hat{m}=24$ and varying $m_{s}$ in Fig. 8 . The same dependence but for $m_{K}^{2}$ is shown in Fig. 9. The large corrections for fit 10 come from the kaon mass. This is shown in Fig. 10(a) where we plot $m_{\pi}^{2}$ with $\hat{m}$ fixed to its physical value and vary $m_{s}$.

The decay constants and ratios are shown as a function of $m_{s}$ at fixed ratio $m_{s} / \hat{m}$ for $F_{\pi}$ in Fig. 10(b), $F_{K}$ in Fig. 11(a) and $F_{K} / F_{\pi}$ in Fig. 11(b)

Let me finally discuss some results of the calculation of $K_{\ell 3}$ [30]. One major point is that the scalar formfactor can be written as

$$
f_{0}(t)=1-\frac{8}{F_{\pi}^{4}}\left(C_{12}^{r}+C_{34}^{r}\right)\left(m_{K}^{2}-m_{\pi}^{2}\right)^{2}+\frac{8 t}{F_{\pi}^{4}}\left(2 C_{12}^{r}+C_{34}^{r}\right)\left(m_{K}^{2}+m_{\pi}^{2}\right)-\frac{8}{F_{\pi}^{4}} t^{2} C_{12}^{r}+\tilde{\Delta}(t) .(4
$$

$\tilde{\Delta}(t)$ contains no $C_{i}^{r}$ and only depends on the $L_{i}^{r}$ at order $p^{6}$. All needed parameters $C_{i}^{r}$ for $f_{+}(0)=$ $f_{0}(0)$ can thus be determined experimentally or from the lattice via the slope and curvature of $f_{0}(t)$.

The dependence of $f_{+}(0)-1$ on the masses is shown in Fig. 12. The left plot shows the three corrections, order $p^{4}$, order $p^{6}$ pure two-loop part and order $p^{6}$ the $L_{i}^{r}$-dependent part as well as the sum of those three. It does not include the contribution from the $C_{i}^{r}$. The right plot shows the same contributions divided by $\left(m_{K}^{2}-m_{\pi}^{2}\right)^{2}$. Both are shown as a function of $m_{\pi}^{2}$ with $m_{K}^{2}$ fixed. Note that in the right plot both order $p^{6}$ corrections are rather flat except near the physical pion mass. 

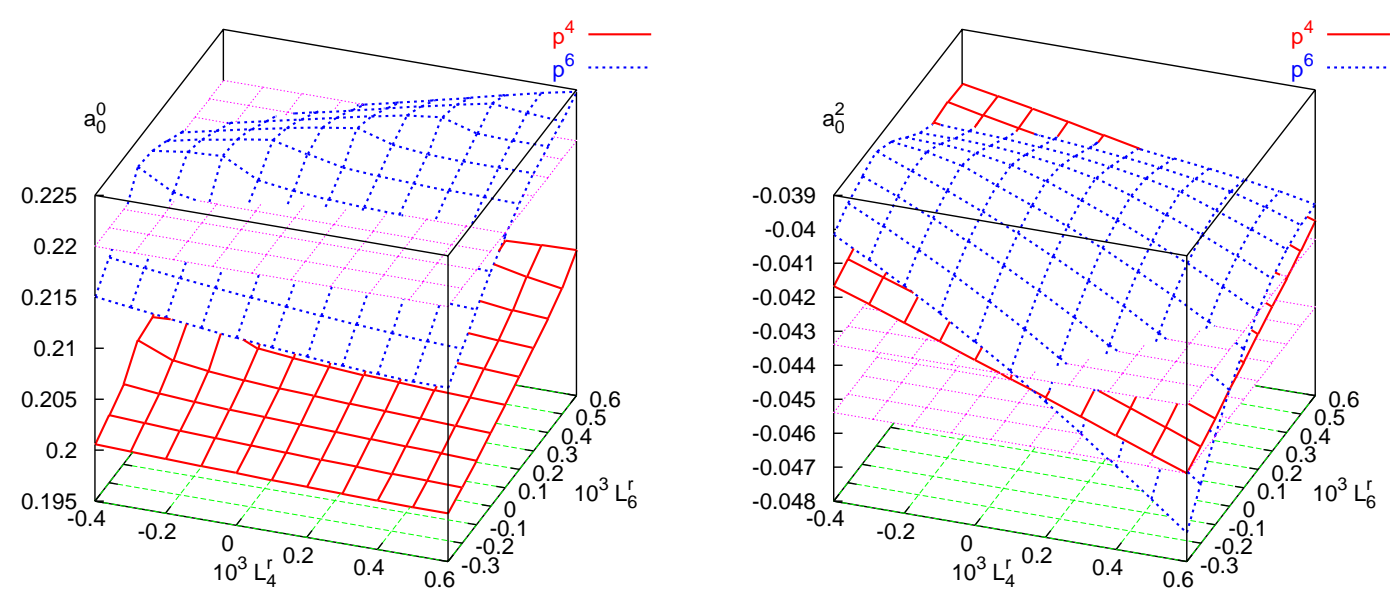

Figure 6: The $\pi \pi$ scattering lengths in three-flavour ChPT as a function of the input values of $L_{4}^{r}, L_{6}^{r}$ used in the fits. $a_{0}^{0}$ left, $a_{0}^{2}$ right. See [48] for details, from [48].
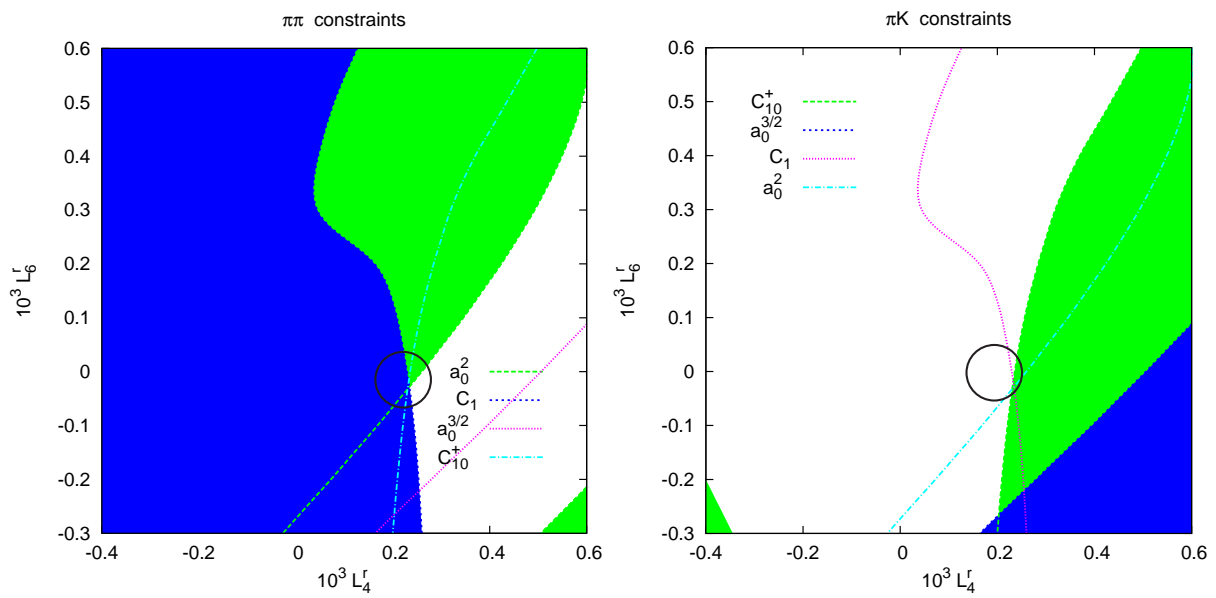

Figure 7: The bounds on $L_{4}^{r}, L_{6}^{r}$ from $\pi \pi$ and $\pi K$-scattering threshold parameters. Left $\pi \pi$ where the bound from $a_{0}^{2}$ shown in Fig. 6 is the most stringent. Right $\pi K$. White regions are allowed. The region of fit $\mathrm{D}$, compatible with both, is indicated by the circle. From [49].

\section{Even more flavours at NNLO (or PQChPT)}

NLO Partially Quenched ChPT has been studied by many people and found to be very useful, see [3] and references therein. The masses and decay constants are known to NNLO for almost all possible mass combinations. Formulas were kept in terms of the quark-mass expansion, analogously to (2.7), to avoid the proliferation in physical masses appearing in this case. The three sea flavour masses and decay constants can be found in $[12,13,67]$ and the two sea flavour results are in [68]. Numerical programs are available from the authors. The formulas are in the papers but can be downloaded from [2]. The papers also contain discussions about how to fit the NNLO LECs. PQChPT NNLO results for neutral masses are in [69] and electromagnetism was included in [70]. 

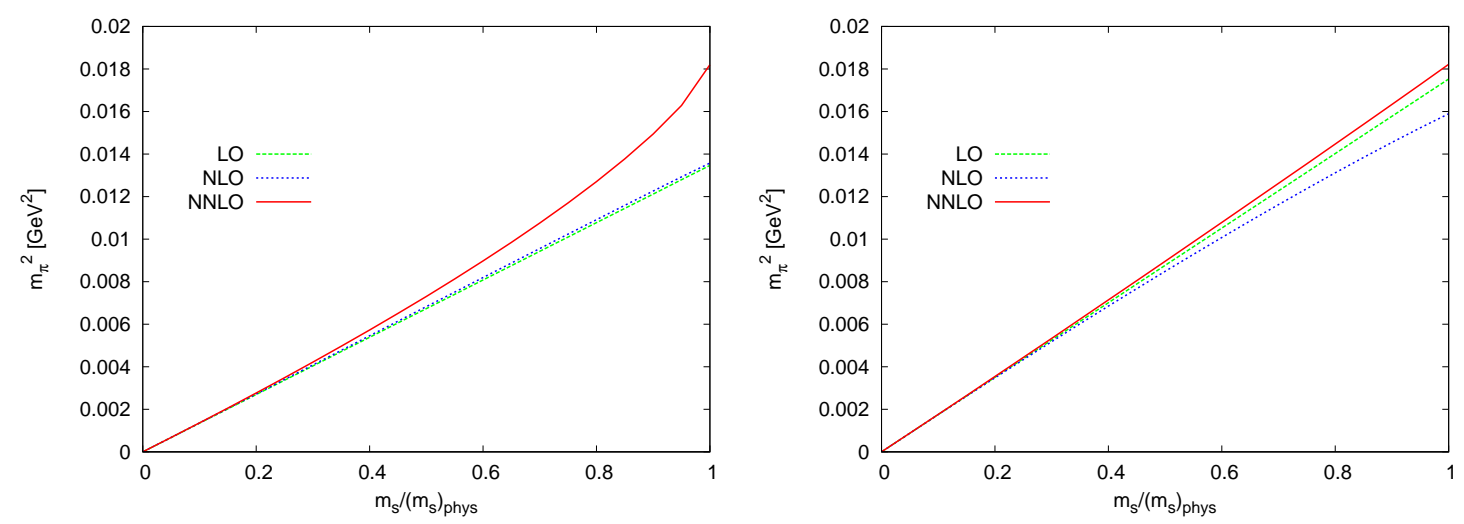

Figure 8: $m_{\pi}^{2}$ as a function of $m_{s}$ for fit 10 (left) and fit D (right) of Tab. 2 with $m_{s} / \hat{m}$ fixed. Note the difference in convergence properties between the two fits.
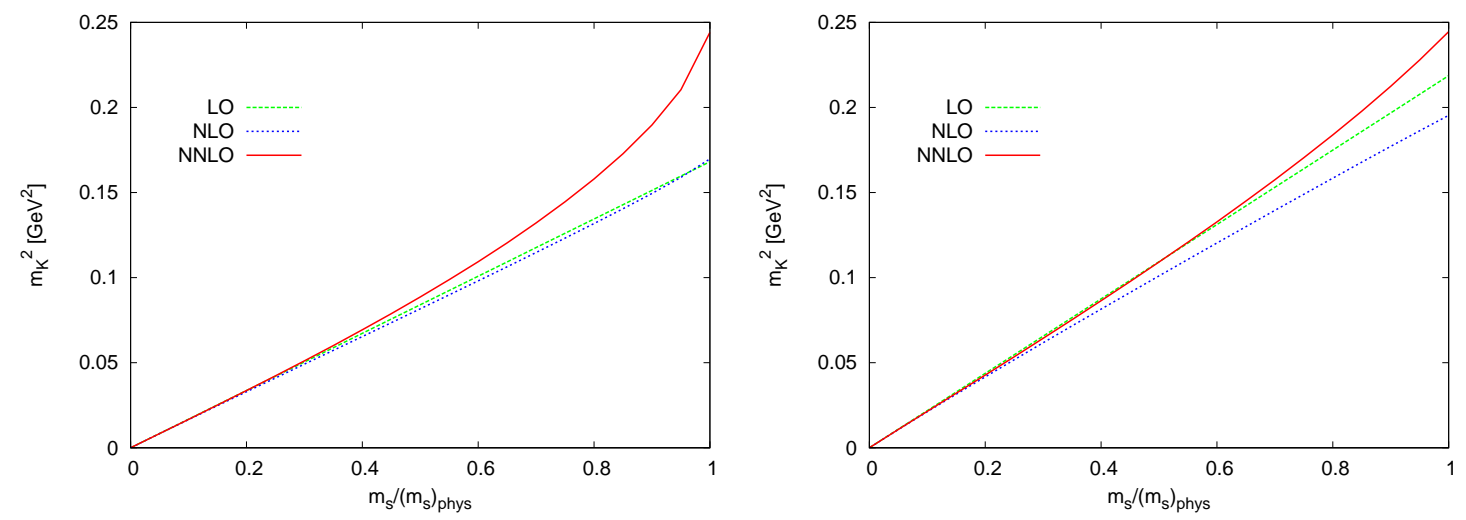

Figure 9: $m_{K}^{2}$ as a function of $m_{s}$ for fit 10 (left) and fit D (right) of Tab. 2 with $m_{s} / \hat{m}$ fixed. Note the difference in convergence properties between the two fits.

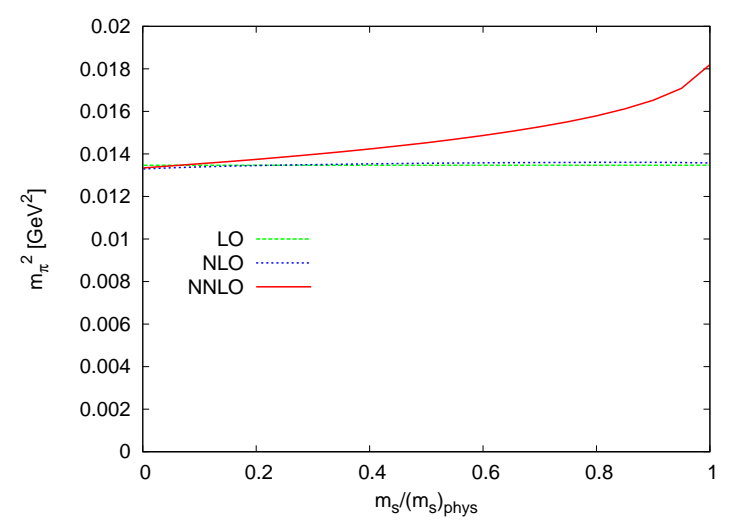

(a)

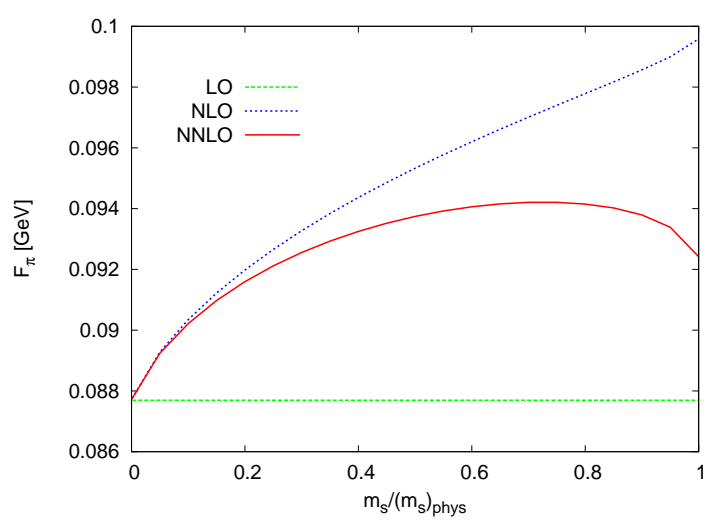

(b)

Figure 10: For fit 10: (a) $m_{\pi}^{2}$ as a function of $m_{s}$ with $\hat{m}$ fixed. (b) $F_{\pi}$ as a function of $m_{s}$ with $m_{s} / \hat{m}$ fixed. 


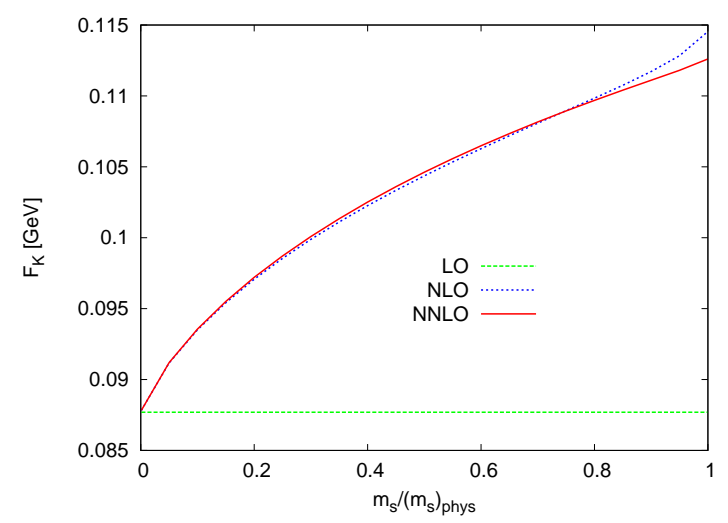

(a)

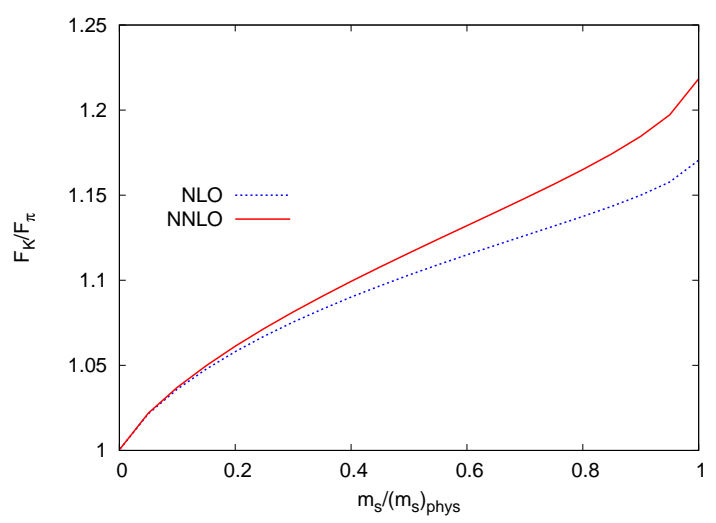

(b)

Figure 11: For fit 10: (a) $F_{K}$ (b) $F_{K} / F_{\pi}$ as a function of $m_{s}$ with $m_{s} / \hat{m}$ fixed.

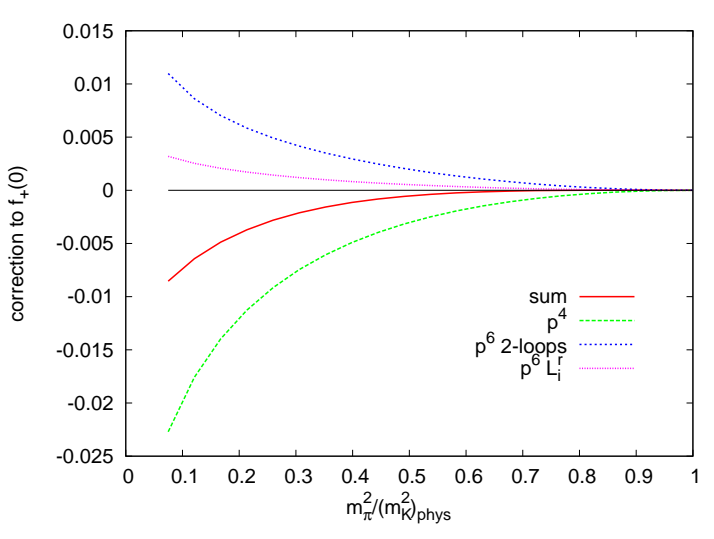

(a)

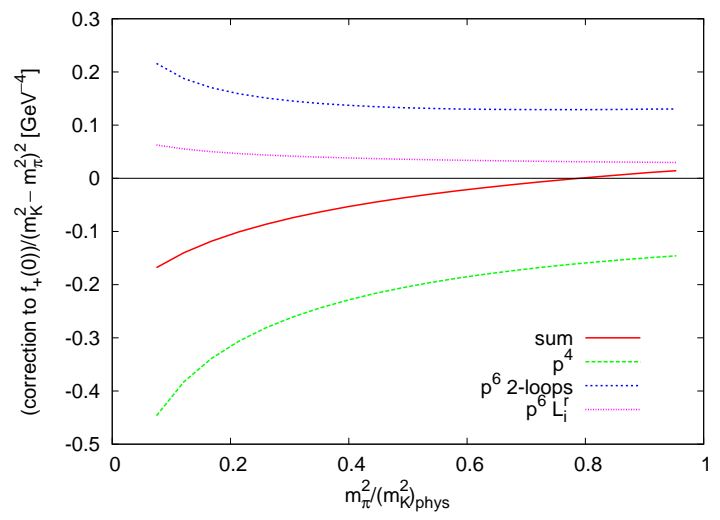

(b)

Figure 12: For fit 10: (a) $f_{+}(0)-1$ (b) $\left(f_{+}(0)-1\right) /\left(m_{K}^{2}-m_{\pi}^{2}\right)^{2}$ as a function of $m_{\pi}^{2}$ with $m_{K}^{2}$ fixed.

\section{Wishing lists and Conclusions}

The conclusions are several lists. First a general wishing list and a comment on NNLO fitting:

- Quark mass dependences everywhere

- A presentation of lattice results at a given quark mass extrapolated to the continuum and infinite volume. Or, in general, results presented in a way that allows us ChPT practitioners to use your data also later on when other inputs might be changed.

- More use of the existing NNLO calculations, if you have any ideas how we can improve the usability of the existing calculations, please tell us. But remember:

- The number of new parameters at NNLO is very small compared to present lattice fitting results. The new order $p^{6}$ parameters are exactly the same in number as those you add when you add an analytic NNLO fitting expression. You therefore do not lose any predictivity when including the full NNLO result and including the known NNLO parts is definitely recommended.

- There is a small caveat to this, the order $p^{4}$ LECs that show up in scattering now appear at NNLO also for the masses and decay constants but these LECs are well known for 
$n_{F}=2, \bar{l}_{1}, \bar{l}_{2}$ from Eq. (3.1), and $n_{F}=3, L_{1}^{r}, L_{2}^{r}, L_{3}^{r}$ from Tab. 2. For the partially quenched case, there is the unknown extra parameter $\hat{L}_{0}^{r}$, but a large $N_{c}$ estimate gives $\hat{L}_{1}^{r}=\hat{L}_{2}^{r}=0$ and thus $L_{0}^{r}=2 L_{1}^{r}, \hat{L}_{3}^{r}=L_{3}^{r}+4 L_{1}^{r}$, (obtained by inverting (21) of [13]).

A more direct wishing list (with input from Berne) for two-flavours is

- $\bar{l}_{3}$ and errors.

- $\bar{l}_{4}$ : can the lattice check the relation between $F_{\pi}$ as a function of the masses and the scalar radius?

- $\bar{l}_{4}$ and [29]: Can the lattice check the strong correlation between the scalar radius and $a_{0}^{2}$ ?

- Isospin breaking in $\pi \pi$ scattering at $s=m_{K}^{2}$, important for CP-violation phenomenology.

A similar list for three flavours (the different points are rather related)

- Large $N_{c}$ suppressed couplings like $L_{4}^{r}$ and $L_{6}^{r}$

- $m_{s}$ dependence of $F_{\pi}$ and $m_{\pi}^{2}$

- sigma terms and scalar radii

- $f_{+}(0)$ in $K_{\ell 3}$ from extrapolations of both $f_{+}\left(q^{2}\right)$ and $f_{0}\left(q^{2}\right)$

and my final comments

- Lots of analytical work is done in ChPT at NNLO, please use (and cite $\odot$ ) it.

- Use the correct ChPT

- 2-flavour for varying $\hat{m}$ and possibly for $N_{f}=2$ and $N_{f}=2+1$ at fixed $m_{s}$ (but have different LECs)

- otherwise 3-flavour

- the various partially quenched versions

- Remember at which order in ChPT you compare things: NLO for both lattice and continuum,

NNLO for both lattice and continuum. Fits put neglected higher order effects into the LECs.

And finally, ChPT and LECs played to my great pleasure a large role in many of the presentations at this conference, as summarized by S. Necco[71]. I am looking forward to even more future results from lattice QCD.

\section{Acknowledgments}

I want to thank the organizers for a most enjoyable meeting, S. Dürr for useful comments on the manuscript, my collaborators and the many people at lattice 2007 I discussed various aspects of lattice QCD and ChPT with.

This work is supported in part by the European Commission RTN network, Contract MRTNCT-2006-035482 (FLAVIAnet), the European Community-Research Infrastructure Activity Contract RII3-CT-2004-506078 (HadronPhysics) and the Swedish Research Council.

\section{References}

[1] J. Bijnens, Prog. Part. Nucl. Phys. 58 (2007) 521 [hep-ph/0604043].

[2] http://www.thep.lu.se/ bijnens/chpt.html 
[3] S. R. Sharpe, hep-lat/0607016.

[4] S. Weinberg, Physica A 96 (1979) 327.

[5] J. Gasser and H. Leutwyler, Annals Phys. 158 (1984) 142.

[6] J. Gasser and H. Leutwyler, Nucl. Phys. B 250 (1985) 465.

[7] H. Leutwyler, Annals Phys. 235 (1994) 165 [hep-ph/9311274].

[8] S. Descotes-Genon, this conference. arXiv:0709.0265 [hep-lat].

[9] S. Weinberg, Phys. Rev. 166 (1968) 1568.

[10] J. Bijnens, G. Colangelo and G. Ecker, J. High Energy Phys. 9902 (1999) 020 [hep-ph/9902437].

[11] H. W. Fearing and S. Scherer, Phys. Rev. D 53 (1996) 315 [hep-ph/9408346].

[12] J. Bijnens, N. Danielsson and T. A. Lähde, Phys. Rev. D 70 (2004) 111503 [hep-lat/0406017].

[13] J. Bijnens, N. Danielsson and T. A. Lähde, Phys. Rev. D 73 (2006) 074509 [hep-lat/0602003].

[14] C. Haefeli, M. A. Ivanov, M. Schmid and G. Ecker, arXiv:0705.0576 [hep-ph].

[15] J. Bijnens, G. Colangelo and G. Ecker, Annals Phys. 280 (2000) 100 [hep-ph/9907333].

[16] J. Gasser and U. G. Meissner, Nucl. Phys. B 357 (1991) 90.

[17] G. Colangelo, M. Finkemeier and R. Urech, Phys. Rev. D 54 (1996) 4403 [hep-ph/9604279].

[18] M. Knecht et al., Nucl. Phys. B 457 (1995) 513 [hep-ph/9507319].

[19] S. Bellucci, J. Gasser and M. E. Sainio, Nucl. Phys. B 423 (1994) 80 [Erratum-ibid. B 431 (1994) 413] [hep-ph/9401206].

[20] J. Gasser, M. A. Ivanov and M. E. Sainio, Nucl. Phys. B 728 (2005) 31 [hep-ph/0506265].

[21] U. Bürgi, Phys. Lett. B 377, 147 (1996) [hep-ph/9602421].

[22] U. Bürgi, Nucl. Phys. B 479 (1996) 392 [hep-ph/9602429].

[23] J. Gasser, M. A. Ivanov and M. E. Sainio, Nucl. Phys. B 745 (2006) 84 [hep-ph/0602234].

[24] J. Bijnens et al., Phys. Lett. B 374 (1996) 210 [hep-ph/9511397].

[25] J. Bijnens et al., Nucl. Phys. B 508 (1997) 263 [Erratum-ibid. B 517 (1998) 639] [hep-ph/9707291].

[26] J. Bijnens, G. Colangelo and P. Talavera, J. High Energy Phys. 9805 (1998) 014 [hep-ph/9805389].

[27] J. Bijnens and P. Talavera, Nucl. Phys. B 489 (1997) 387 [hep-ph/9610269].

[28] G. Colangelo and C. Haefeli, Nucl. Phys. B 744 (2006) 14 [hep-lat/0602017].

[29] G. Colangelo, J. Gasser and H. Leutwyler, Nucl. Phys. B 603 (2001) 125 [hep-ph/0103088].

[30] J. Bijnens and P. Talavera, Nucl. Phys. B 669 (2003) 341 [hep-ph/0303103].

[31] P. Post and K. Schilcher, Eur. Phys. J. C 25 (2002) 427 [hep-ph/0112352].

[32] G. Amorós, J. Bijnens and P. Talavera, Nucl. Phys. B 568 (2000) 319 [hep-ph/9907264].

[33] E. Golowich and J. Kambor, Nucl. Phys. B 447 (1995) 373 [hep-ph/9501318].

[34] S. Dürr and J. Kambor, Phys. Rev. D 61 (2000) 114025 [hep-ph/9907539].

[35] K. Maltman, Phys. Rev. D 53 (1996) 2573 [hep-ph/9504404]. 
[36] B. Moussallam, J. High Energy Phys. 0008 (2000) 005 [hep-ph/0005245].

[37] J. Bijnens, unpublished.

[38] E. Golowich and J. Kambor, Phys. Rev. D 58, 036004 (1998) [hep-ph/9710214].

[39] G. Amorós et al., Nucl. Phys. B 602 (2001) 87 [hep-ph/0101127].

[40] G. Amorós et al., Phys. Lett. B 480 (2000) 71 [hep-ph/9912398].

[41] G. Amorós et al., Nucl. Phys. B 585 (2000) 293 [Erratum-ibid. B 598 (2001) 665] [hep-ph/0003258].

[42] J. Bijnens and K. Ghorbani, Phys. Lett. B 636 (2006) 51 [hep-lat/0602019].

[43] P. Post and K. Schilcher, Phys. Rev. Lett. 79 (1997) 4088 [hep-ph/9701422].

[44] P. Post and K. Schilcher, Nucl. Phys. B 599 (2001) 30 [hep-ph/0007095].

[45] J. Bijnens and P. Talavera, J. High Energy Phys. 0203 (2002) 046 [hep-ph/0203049].

[46] C. Q. Geng, I. L. Ho and T. H. Wu, Nucl. Phys. B 684 (2004) 281 [hep-ph/0306165].

[47] J. Bijnens and P. Dhonte, J. High Energy Phys. 0310 (2003) 061 [hep-ph/0307044].

[48] J. Bijnens, P. Dhonte and P. Talavera, J. High Energy Phys. 0401 (2004) 050 [hep-ph/0401039].

[49] J. Bijnens, P. Dhonte and P. Talavera, J. High Energy Phys. 0405 (2004) 036 [hep-ph/0404150].

[50] J. Gasser, C. Haefeli, M. A. Ivanov and M. Schmid, arXiv:0706.0955 [hep-ph].

[51] R. Kaiser, arXiv:0707.2277 [hep-ph].

[52] R. Kaiser and J. Schweizer, J. High Energy Phys. 0606 (2006) 009 [hep-ph/0603153].

[53] J. Bijnens and K. Ghorbani, work in progress.

[54] J. Bijnens, arXiv:0707.0419 [hep-ph].

[55] J. Bijnens and T.A. Lähde, work in progress

[56] J. Gasser, C. Haefeli, M. A. Ivanov and M. Schmid, work in progress

[57] G. Ecker, J. Gasser, A. Pich and E. de Rafael, Nucl. Phys. B 321 (1989) 311.

[58] M. Knecht and A. Nyffeler, Eur. Phys. J. C 21 (2001) 659 [hep-ph/0106034];

[59] S. Peris, M. Perrottet and E. de Rafael, J. High Energy Phys. 9805 (1998) 011 [hep-ph/9805442].

[60] J. Bijnens et al., J. High Energy Phys. 0304 (2003) 055 [hep-ph/0304222].

[61] V. Cirigliano et al., Nucl. Phys. B 753 (2006) 139 [hep-ph/0603205].

[62] S. Pislak et al. [BNL-E865 Collaboration], Phys. Rev. Lett. 87 (2001) 221801 [hep-ex/0106071].

[63] S. Pislak et al. [BNL-E865 Collaboration], Phys. Rev. D 67 (2003) 072004 [hep-ex/0301040].

[64] J. Bijnens and J. Prades, Nucl. Phys. B 490 (1997) 239 [hep-ph/9610360].

[65] J. Bijnens, N. Danielsson and T.A. Lähde, hep-ph/0701267, to be published in Acta. Phys. Pol. B.

[66] P. Büttiker, S. Descotes-Genon and B. Moussallam, Eur. Phys. J. C 33 (2004) 409 [hep-ph/0310283].

[67] J. Bijnens and T. A. Lähde, Phys. Rev. D 71 (2005) 094502 [hep-lat/0501014].

[68] J. Bijnens and T. A. Lähde, Phys. Rev. D 72 (2005) 074502 [hep-lat/0506004].

[69] J. Bijnens and N. Danielsson, Phys. Rev. D 74 (2006) 054503 [hep-lat/0606017].

[70] J. Bijnens and N. Danielsson, Phys. Rev. D 75 (2007) 014505 [hep-lat/0610127].

[71] S. Necco, this conference. 